\title{
EngineERs-IN-RESIDENCE Programs AS A FrameWORK FOR INDUSTRY ENGAGEMENT IN UNDERGRADUATE ENGINEERING EDUCATION: CHALLENGES AND OPPORTUNITIES
}

\author{
Marcia Friesen ${ }^{1}$, Nadine Ibrahim ${ }^{2}$, Grant McSorley ${ }^{3}$, and Stephen Mattucci ${ }^{4}$ \\ ${ }^{1}$ University of Manitoba, ${ }^{2}$ University of Waterloo, ${ }^{3}$ University of Prince Edward Island, and ${ }^{4}$ McMaster University \\ Corresponding author marcia.friesen@umanitoba.ca
}

\begin{abstract}
Industry engagement in undergraduate engineering education is a community-centred approach to learning that is hands-on and links the engineering theory to practice. This paper provides a review of existing Engineer-in-Residence (EIR) programs in Canada, including the University of Manitoba, Dalhousie University, University of Calgary, Ryerson University, University of Ottawa, and the University of Waterloo, as well as a brief international scan. We consider the motivations behind the institutions' initiative to introduce EIR programs, different types of engagements, challenges, and opportunities. Programs are also examined externally relative to professional residency programs in business schools, among others, and relative to other forms of industry engagement in undergraduate engineering education. A brief overview of the history and role of EIRs within engineering programs is also presented. The paper will be of interest to those exploring a similar industry engagement framework at their institution, and offers a forward-looking perspective on ways to leverage the skills and experience of practicing engineers in preparing students to tackle the challenges of the future.
\end{abstract}

Keywords: Engineers-in-Residence, industry engagement, engineering practice, professionalism

\section{INTRODUCTION}

In order to keep pace with the rapid technological advancements impacting society, it is increasingly more necessary for our approaches to educate the engineers of tomorrow to be integrated in real-world contexts [9]. Further, most engineering faculty have followed traditional academic pathways into their roles, and therefore are often not knowledgeable of industry best practices [5]. Engineerin-Residence (EIR) programs can provide a direct link to real world industry exposure within curricular programming. EIR programs and positions have been established at various institutions around the world as a mechanism to provide role models to students, bridge the divide between university and engineering practice, help students secure work placement opportunities, provide mentorship to student teams, introduce legislation and professional engineering societies to students, and ultimately help develop engineering graduates who are better prepared to enter the workplace [5], [6], [8], and [9]. The programs are ultimately driven when the institution sees industry engagement as a critical part of the culture and making the engineering program successful [5]. However, the roles and responsibilities of EIRs vary widely based on context and the host institution.

The benefits that an EIR may provide to a faculty have been reported to be greater than expected when the role was first envisioned, as EIRs are more involved in teaching, with different perspectives on course content (such as the expansion of the design process), and different approaches to mentorship of students [5] and [9]. Further, students perceive a general increase in credibility of the course content [9]. EIRs are also well equipped to help students understand the value of important, but often overlooked professional skills such as: teamwork, communication, professional ethics, etc. [6] and [8]. EIRs may also be responsible for developing and fostering relationships with stakeholders from industry, community, and government, including: building case studies, and organizing site visits and guest speakers [5]. Benefits are multi-directional, as EIRs recent academic experiences allow them to better relay on-campus needs and limitations back to industry colleagues [9].

This paper examines several approaches to integrating a practice-based perspective in undergraduate engineering education, mostly notably through Engineers-in-Residence (EIR) programs that exist at several universities. EIR programs are one way to foster a diversity of perspectives in the education of future engineers for both faculty and students. As professional engineers with considerable industry experience (generally 15+ years), EIRs contribute at a professional but non-professorial rank in engineering faculties, communicating and demonstrating the practice community's needs and expectations relative to knowledge, skills, and values of graduating engineers. They enhance curriculum with current practice-based 
expertise, approaches, bodies of knowledge, and skills for ongoing curriculum innovation and relevance. This can occur in a variety of contexts including teaching or coteaching courses and/or labs, design competition team mentorship, capstone design advising, and other opportunities in which EIRs have an opportunity to shape engineering students' knowledge, skills, and values for entry to practice. They can also support student recruitment post-graduation to mutually benefit both students and employers. Likewise, the practice community can benefit when EIRs share an increased understanding of the University's mandate and the Faculty of Engineering's scope of activities in teaching \& learning, research, and service back to the practice community.

\subsection{Background of Industry Engagement in Academia}

Originally, engineering was a profession learned through apprenticeship models of training, and then sought a place in universities as a way to define a consistent body of knowledge and scope of application, and to afford status to the profession. After World War II, university education in engineering became decidedly more theoretical, focused on engineering science, in response to economic and political priorities in national defense in North America, and this trend continued for many decades. In the 1980s, a renewed emphasis on design became a consistent theme, often defined as a return to the hands-on, practice-based origins of the profession. Since that time, curricula have expanded ways to balance a theoretical basis with a practice-based perspective, to ensure that graduate engineers enter the profession with the appropriate balance of know-what (theory) and know-how (practice).

\subsection{Theoretical and Conceptual Frameworks}

The authors believe the Engineer-in-Residence programs adopt a community-centered perspective on student learning from the How People Learn (HPL) Framework [2]. The community-centered framework is presented as an approach which provides connections to the outside world, to support core learning values, and develops norms for both the classroom and school which encourage collaboration and learning. The connections to the outside world are obvious as EIRs are typically defined individuals with extensive industry experience.

The distributed cognition theory (within communitycentered framework) applies at both the classroom (i.e. student) and institutional level, where the community is comprised of a combination of perspectives and expertise - where the EIR is unique in both cases. Within classroom learning environments, EIRs are able to provide to the student body a source of unique perspectives rooted in practice-based experience that are otherwise not readily available. This may occur through direct classroom instruction, project supervision, or even through secondary peer-to-peer interactions. EIRs also provide further perspectives, support, and resources within the faculty and staff community, and model the importance of diverse skills and experiences within the institution.

To more deeply explore the learning that occurs at the student level, employer engagement in education can be conceptualized through the 'life cycle' theory, where life is contextually constructed through experiences [7]. The basis of this framework and the benefits of employer engagement can be categorized into human, social, and cultural capital. Human capital relates to the education or training itself - the skills that contribute to an individual's employability. Social capital relates to the relationships between people which can be leveraged as a resource one's network. Cultural capital relates to understanding of the social and cultural constructs and pathways - one's knowledge of the system. Mann, et al. describe this framework in the context of work experience placements, but we would argue the same benefits are applicable when industry is brought into the classroom [7]. These experiences are not as deep as when a student is embedded within industry (on a co-op placement for example), however, an Engineer-in-Residence can bring these experiences to the classroom to more widely share with a greater quantity of students, potentially giving opportunities to more students to learn from a breadth of experiences.

Each of the authors are from different institutions, and bring individual motivations related to Engineer in Residence programs. Dr. Marcia Friesen is Director of the Centre for Engineering Professional Practice \& Engineering Education at the University of Manitoba, an early-adopter institution of EIR programs. The University of Manitoba currently has seven active EIRs and an equal number of former / retired EIRs. Dr. Nadine Ibrahim is the Turkstra Chair in Urban Engineering, a new role in the Department of Civil and Environmental Engineering at the University of Waterloo, with a mandate to bring real world municipal engineering leadership scenarios into the classroom, and the selection and oversight of what is envisioned as the Douglas Wright Engineer-in-Residence program. Dr. Grant McSorley is an assistant professor in the Faculty of Sustainable Design Engineering at the University of Prince Edward Island (UPEI), where his interest into EIRs and surveying current practices stems from discussions with colleagues regarding implementing an EIR program at UPEI and the format that would take. Specifically, UPEI is interested in lessons learned from other engineering faculties about what worked well and what did not in terms of roles within the program, interaction with students and faculty, and recruiting EIRs. In the context of UPEI, students, especially in years 3 and 4 , have quite a lot of contact with industry clients (usually an hour every week or two), but these are not always engineers. While there are some engineers on staff who can support projects, these are permanent positions with 
specific roles in the department, so it is not the same as consulting with a practicing engineer. There is an expectation that with an EIR program, the gap can be filled in students' understanding of the practice of engineering and some of the professional components that are difficult to teach, as well as help faculty stay up to date with current industry practices. Dr. Stephen Mattucci is the National Coordinator of the Canadian Engineering Education Challenge (CEEC), an initiative with goals including: supporting cross-institutional collaborations between engineering educators with alignment between priorities, and strengthening the connection between industry and engineering curriculum. This work is the culmination of a discussion that occurred at a CEEC workshop designed to promote collaborations (Project Buildathon, Dec. 2018).

\subsection{Motivation}

This paper seeks to perform a scan of the EIR programs in the Canadian system, an overview of other international EIR programs, in addition to drawing parallels with other similar -in-Residence programs as they are implemented in other disciplines and faculties, as well as highlight program implementation pathways, common challenges faced, and opportunities for further innovation.

\section{METHODOLOGY}

The methodology for information gathering started with a review of EIR programs at the authors' own institutions, followed by an online review of programs both in engineering and externally, and supplemented with select interviews. The interview questions were generally themed to include the motivation behind the development of EIR programs, with explicit and implicit goals, if any (Table 1.). The authors' were interested in what a "typical" EIR role involved and the nature of their roles and activities. From a program development perspective, questions focused on the major milestones in developing such programs, and the enabling features in developing the program (e.g. available funding, support of senior leadership, and finding the right candidate), and the disabling elements, or major barriers faced (and overcame) in developing the programs. The students' response to the EIR programs was also of interest, both from an educational value, and from a career-preparedness perspective. From a logistical perspective, the start of the program, duration of engagement, and number of EIRs, was asked, along with the nature of their engagement (e.g. teaching, outreach, mentorship, advisory, etc.)

Table 1. Questions for EIR Interviews

1. What was the motivation behind the development of the EIR program?

2. What are the explicit and implicit goals of the EIR program?
3. What does a "typical" EIR look like in terms of role and activity at your institution?

4. What were some of the major milestones in developing the program? Also, what were enabling features in developing the program (e.g. available funding, support of senior leadership, the right person at the right time appeared, etc.)

5. What were some of the major barriers you overcame when developing the program? I.e. what were disabling elements in developing the program?

6. If you had to develop an EIR program from scratch, is there anything you would do differently?

7. How have students responded to the EIR program?

8. Logistical details about the EIR program: When did it start? Which departments? Courses involved? Other engagements in the department? How many EIRs are hired at the moment, and since the beginning of the program? Duration of engagement?

\section{REVIEW OF ENGINEERS-IN-RESIDENCE INITIATIVES}

\subsection{Examples from Canada}

\subsubsection{University of Manitoba}

The EIR Program at the University of Manitoba was established in 2001 with Canada's first EIR. Of note, it was a key initiative within the University's then-newly awarded NSERC Chair in Design Engineering program, which provided opportunities for non-traditional initiatives that support undergraduate engineering education. Fundamentally, the EIR Program is designed to offer an avenue for mutually-beneficial engagement between the Faculty of Engineering and the engineering practice community.

Engaging EIRs is one way that the Faculty of Engineering fosters a diversity of perspectives in the education of future graduate engineers. For engineering students and faculty members, EIRs communicate and demonstrate the practice community's needs and expectations relative to knowledge, skills, and values of graduating engineers. EIRs can enhance the curriculum with current practice-based expertise, approaches, bodies of knowledge, and skills for ongoing curriculum innovation and relevance. At the University of Manitoba, this occurs in a variety of contexts including teaching or co-teaching courses and/or labs, design competition team mentorship, capstone design advising, and connecting industry and faculty members for applied research collaboration. EIRs also serve as liaisons to specific industry sectors, by which they can support student hiring to mutual benefits for students and employers, and an increased understanding of the Faculty of Engineering's 
mandate and scope of activities in teaching \& learning, research, and service back to the practice community.

Currently, there are seven active EIRs at the University of Manitoba, and seven former EIRs who have moved into retirement or other activities. Typically, EIRs are registered professional engineers (P.Eng.) in good standing with the provincial regulator with a minimum of 10 years of experience in professional engineering practice. The EIR supplements existing expertise in the Faculty, and does not displace or relieve existing faculty members. Most of the EIR positions are part-time, and to date, many EIRs have included individuals who are in the senior stages of their career and are interested in a part-time commitment as part of a transition to retirement, as well as mid-career engineers who are interested in work-life balance.

Administratively, EIRs are appointed in the Centre for Engineering Professional Practice \& Engineering Education, a cross-faculty unit that coordinates a number of courses and initiatives that cross departmental boundaries, and whose mission includes facilitating educational collaboration with industry. To support EIRs' familiarization to and understanding of the academic environment and academic responsibilities, EIRs are paired with a faculty mentor with whom they will meet regularly for feedback and mentorship on their duties. To support EIRs' success in teaching (where duties include teaching), EIRs are asked to avail themselves of teaching workshops as preparation and ongoing development of teaching proficiency, which are available on campus at no cost.

In their work, EIRs are responsible jointly to the Director of the Centre for Engineering Professional Practice \& Engineering Education and the Head of the department(s)/Program(s) in which the EIRs' activities are housed or delivered. Currently, five of seven EIRs have mechanical engineering backgrounds, with searches ongoing for a Biosystems EIR and an Indigenous EIR. Presently, the majority of EIRs are engaged with external financial sponsorship from sector-specific stakeholders, where these relationships are carefully navigated with the Office of the Dean within the broader context of the Faculty's partnerships with the practice community [14].

\subsubsection{Dalhousie University}

The EIR program at Dalhousie University was started in 2015 and is under the responsibility of the NSERC Design Chair. Over this time, it has hosted nine EIRs of which there are currently four across the five engineering departments. EIRs are under term contracts of typically one year, which has proven convenient in allowing the circulation of engineers between industry and academia. The development of the program was motivated by factors including the limited direct exposure of students to industry and practicing engineers, to allow for more mentoring than was possible via their capstone and coop programs, and the limited industry experience of faculty. The EIR program was also intended to build a network of connections with industry, particularly with local partners, to integrate teaching and research practice with industry practices, and to have the EIRs bring their network and expertise to the university. The program was also influenced by the work of Ron Britton at the University of Manitoba.

EIRs are hired on a part time, $20 \%$ basis and provided with a stipend of approximately $\$ 20,000$, which is currently funded through the NSERC Design Chair. In terms of career path, currently most EIRs are mid-career, either returning to practice after parental leave or consulting engineers who have flexibility to take on the part time position.

The three main activities of EIRs at Dalhousie University are:

1. Mentoring students regarding design problems. This mainly takes place during capstone projects, but there is a drive to expand this to other years. Large cohort sizes (e.g. 450 first year students) add complexity to this. While EIRs will attend some classes, they do not take on formal teaching responsibilities.

2. Connecting with industry: This is primarily the identification of capstone projects and making connections with new companies.

3. Managing special events: Primarily the Dalhousie Capstone Conference for which EIRs are the project managers and which includes over 50 industry judges plus sponsors.

Critical factors to a successful EIR program have been found to include a common context or discipline with students and faculty, the involvement of the department in the hiring process, and being a current fit with the existing EIR team.

If there is little overlap between the EIR's expertise and projects or courses in which they are involved, student and faculty engagement can be negatively impacted. Similarly, it was found that involving the department in the hiring process, rather than placing responsibility with the Dean, helped to ensure faculty buy in and the integration of the EIR as part of the department. Lastly, as the EIRs also work as a team, it is important for new hires to fit with existing participants.

Students in capstone design courses are required to meet with EIRs for an initial 15-minute meeting at the beginning of the semester and it is then left up to the students to decide on their level of engagement. EIRs will also attend some classes. Approximately 50\% of students return for follow up consultations, and of those, nearly all consult with the EIRs on a regular basis. The faculty have found that the benefits of EIRs to student development include the development of 'soft', or permanent, skills such as teamwork, client relationship, research skills and dealing with "wicked" problems. 
While there are no explicit targets or outcome assessments for EIRs, a Senate Review by external experts recognised the initiative as an area of excellence to be promoted.

A main barrier to the implementation of the program is the funding source, and a key recommendation is to have a sustainable source of funds in place before starting and EIR program. While it would be useful to have increased contact hours either through more EIRs or increasing the hours per week, they do not believe that they could engage an EIR on a full-time basis.

\subsubsection{University of Calgary}

A rather unique approach to the EIR program was started at the University of Calgary with the hiring of their first EIR ${ }^{1}$, at the Schulich School of Engineering, who is also the first indigenous EIR in Canada, and self identifies as an Indigeneer ${ }^{\mathrm{TM}}$. The indigenous EIR program was initiated as a pilot project, which was funded through a grant in response to the University's Indigenous Strategy [12]. She was hired as a consultant to operationalize Indigenous inclusion and the Truth and Reconciliation Commission Calls to Action. Since 2017, EIR involvement included curriculum development to recognize indigenous innovation and support existing students and faculty. For example, the EIR has been working on an initiative to Indigenize pipeline curriculum through a pilot project with SAIT and Innotech Alberta, training 60 Indigenous students in Pipeline Monitoring. Another example includes starting an Indigenous Student Club to build awareness and develop STEM capacity for indigenous students, together with students at Mount Royal University. She is contracted for a once-a-month commitment, though she finds that she goes beyond that, driven by a duty of care. A second EIR (non-indigenous) recently started at the University of Calgary in early 2019. Both EIRs fall under the responsibility of the NSERC Chairs in Design Engineering.

\subsubsection{Ryerson University}

The EIR program at Ryerson University is primarily an outreach role, specifically designed to increase the enrolment of women in engineering, and improve their engineering education experience. As an industrysponsored initiative which started in 2012, Hydro One's Women in Engineering program supports a consortium of four universities in Ontario: Ryerson University, University of Waterloo, Western University, and the University of Ontario Institute of Technology (now Ontario Tech). At Ryerson University, the EIR program is focused on Electrical Engineering, a program with historically low rates of female enrolment. For the past three years, a female director of Hydro One is the $\operatorname{EIR}^{2}$ at Ryerson University, where the EIR participates in events

\footnotetext{
1 Deanna Burgart, P. Eng is the first EIR at the University of Calgary.
}

as a motivational speaker, and serves as a mentor to young women [26].

\subsubsection{University of Waterloo}

Also part of the same Hydro One program, a female engineer is the $\operatorname{EIR}^{3}$ at the University of Waterloo in Electrical Engineering [1]. The program is led by the corporate culture of promoting and managing diversity, and stems from Hydro One's overall Diversity and Inclusion Program. She is considered the industry representative in the department of Electrical Engineering, and commits 5-8 business days annually towards keynote events and offers her technical expertise. There is a rotation at Hydro One for the EIR selection and involvement, and is considered a way to promote women in leadership and an effort towards community engagement.

\subsubsection{University of Ottawa}

The Department of Civil Engineering at the University of Ottawa has its own Engineer-in-Residence, who serves as a liaison between industry and academia [17]. An example of his role involves inviting engineers from industry to suggest useful projects and to serve as mentors for $4^{\text {th }}$ year students" "capstone projects" [18]. In the School of Electrical Engineering and Computer Science, the NSERC Chair in Entrepreneurial Engineering Design is the Engineer-in-Residence, and is also the faculty coordinator in entrepreneurship and innovation. In their roles as NSERC Chair, the EIR oversees client-based design projects, and invites industry to sponsor projects [19]. A private donation over five years allowed the University of Ottawa to hire a full-time engineer-inresidence to guide and coach the extra-curricular student design competition teams.

\subsection{International Examples}

The specific goal at Ohio North University is to bring the professional workplace into the academic environment [6]. This is achieved through an EIR, who is employed and funded by an industry partner organization (Marathon Ashland Petroleum LLC), but hosted at the university with a dedicated office, and a suite of student cubicles. The program enables students work-time on industry projects while on campus, under the direction of the EIR. The balance of courses and workplace projects helps to develop student time management skills. The university benefits from strong connections to a regional and national industry partner, which also helps to support accreditation. Industry benefits from the help in completing projects, and is exposed to soon-to-be graduates. The EIR program also serves a strong recruitment tool, as students are able to gain industry experience, without taking an additional year for a co-op placement [6].

\footnotetext{
${ }^{2}$ Lyla Garzouzi, P. Eng is the EIR at Ryerson University.

${ }^{3}$ Gordana Andjelkovic, P. Eng. Is the EIR at the University of Waterloo.
} 
The EIR program at Colorado State University brings engineering professionals to campus as volunteers to interact with students within laboratory environments [8]. Students see significant value in their increased face-toface exposure to industry, and EIRs find value in giving back to the community and potentially recruiting promising graduates [8].

The EIR role at Charles Sturt University, Australia, was initially envisioned to provide role models for student engineers, however would more broadly expand to developing a culture of industry engagement amongst all staff and student engineers [5]. Terms are fixed periods of time to ensure EIRs maintain relevant industry experience and knowledge, which must be demonstrated in order to reapply to continue a position. Three staff are employed as EIRs at Charles Sturt University engineering, and are involved in teaching courses, mentoring student teams, and curriculum development by actively seeking industry input on the desired skills and attributes of future graduates. The EIR program has identified future possibilities for expansion by allowing EIRs to undertake outside consulting work, benefitting the program by populating fresh resources for case studies and projects, as well as financial gain, and keeping EIRs continually involved in relevant industry experiences [5].

\section{EXAMPLES FROM OTHER DISCIPLINES}

\subsection{Management and Business}

Business schools and schools of management sciences have long created the connection to business leaders and the corporate community, and lend themselves naturally to programs such as Executives-In-Residence and Entrepreneurs-In-Residence.

At York University, the Executive-in-Residence at the School of Public Policy and Administration, in the Faculty of Liberal Arts and Professional Studies, is a role that is grouped together with individuals who serve as advisory members and visiting professors [25]. Executives-InResidence at the Schulich School of Business are a long list of professionals who, together with the advisory council, serve in the Dean's office for industry liaison and support.

At the Rotman School of Management at the University of Toronto, industry ties help keep the school up-to-date [22]. Local business leaders are invited to participate in the Executive-In-Residence program, where they engage with students, staff, and faculty by ways of providing guest lectures, mentoring students, advising student groups of projects, sitting on advisory boards and/or teaching in executive non-degree programs. Some also have Adjunct Professor titles, and teach courses for-credit. Rotman Commerce has also started their own Executive-InResidence program, offering guidance to students in their executive and entrepreneurial endeavours [23].

The University of Manitoba has several other professional residencies that share common attributes with its Engineer-in-Residence program. Executives-inResidence at the Asper School of Business are individuals with a proven track record in business, and engage with students and faculty in multiple areas. Representative activities including developing relationships with business sector councils and professional bodies, as well as developing internship opportunities with the Manitoba business community, teaching courses as sessional lecturers, coaching and mentoring of students in case competitions and graduate students in industry consulting projects [13].

\subsection{Architecture and Planning}

Also at the University of Manitoba, the Faculty of Architecture houses a Partner-In-Residence (PIR) program, which advances thinking and practice in the investigation of the critical design issues facing Manitoba's built environments, Northern communities and design culture [15]. A primary goal of the Partner-In-Residence role is to shape initiatives, which will bridge the interests, needs, and aspirations of students, academics, professional designers, industry and the public focussed on the livability and community development challenges of the region [15]. Further, the School of Art offers six-month residency for an Indigenous Designer, who interacts closely with students and faculty. As an active and engaged interdisciplinary designer, they produce a body of new creative work and research to extend the collective understanding of design. They also engage in courses to introduce new ways of thinking, new perspectives and new knowledge [16].

At the University of Waterloo's School of Planning, for almost 25 years, the school hosts a Planner-In-Residence annually, to teach and conduct seminars. The Planner-inResidence program is funded by the University of Waterloo Planning Alumni of Toronto [24]. The value of this program is in leveraging the experience and skills of professionals who are leaders in their field, both to the benefit of students and faculty who have the opportunity to interact with them.

\subsection{Information and Library Sciences}

The Libraries at the University of Toronto hired its first "Wikipedian" in Residence, in the 2018/2019 academic year. A Masters Candidate in the Department of Political Science has been brought on to participate in a range of activities, including researching collections and articles to be shared with the Wikipedia community; adding content to the Wikimedia Commons and Wikisource sites from the university's special collections; and introducing strategies to improve the university libraries' Wikipedia presence, and promoting participation in new or existing Wiki projects related to the holdings of the libraries [20]. The Wikipedian in Residence also organizes special events, such as editing challenge days and training workshops. Following suit, Concordia University also 
hired a Wikipedian in Residence with a goal of fostering digital literacy among the faculty and students [4]. Through a commitment of 6-8 hours per week to the residency, the Wikipedian in Residence has similar roles, in addition to assisting instructors and librarians in integrating Wikipedia into their teaching and learning activities, and coordinating edit-a-thons to bring Wikipedians on campus to work with faculty and staff to create and improve Wiki content.

\subsection{Literature and Creative Writing}

Bringing the real world of writing into creative writing classes, a Writer-in-Residence program was started in 2013 at the University of Toronto's Department of English for those enrolling in a creative writing minor. The interaction of the students with the Writers-in-Residence, who are accomplished writers in their fields, involves regular office hours, in-class workshops, and creative writing groups [23]. The Writers-in-Residence also put on reading performances and deliver keynote lectures to the student body.

\section{CHALLENGES AND OPPORTUNITES}

The EIR programs are introduced as an academicindustry engagement, and take on various organizational frameworks from their inclusion as advisory or outreach to an immersion into the core-teaching mandate at universities. With EIR involvement on- or off-campus, lasting one term or one year or more, their effectiveness stems from their reason for being, perhaps in response to fulfilling a technical gap, the need to bring in indigenous perspectives in curricula, or improving the enrolment of women in engineering.

Depending on the explicit role of the EIR, when a person is positioned within an academic institution, they may no longer be immersed in industry, and risk losing the perspective that adds so much value [5]. Some institutions have counteracted this with only part-time or casual appointments, or fixed term employment to ensure that EIRs maintain relevant industry knowledge and experience [5], or recruiting experienced, retired engineers who were keen to return some of their expertise to the future of the profession [9].

Often one of the criteria for an EIR is someone with extensive industry experience, which may be accompanied by high salary expectations [5]. To overcome financing challenges, there have been instances of industry partners collaboratively funding a position that directly benefits their industry sector, for example an Aerospace EIR in Manitoba [9]. Depending on how the EIR program is integrated within the curriculum or faculty/department, not all students are exposed to an EIR or have the opportunity to work with an EIR [8].

At Dalhousie University, University of Manitoba, and University of Calgary, the EIR Program grew out of the opportunities afforded by the NSERC Chairs in Design
Engineering program. The Chairs in Design Engineering program will no longer be offered by NSERC, as NSERC replaces many of its partnership programs with a new research partnerships program, NSERC Alliance Grants. At time of writing, there has not been any experience with the NSERC Alliance Grants to understand whether and how they may support EIR-style engagements, and the extent to which other funding models will need to be envisioned.

\section{RECOMMENDATIONS FOR EIR PROGRAM IMPLEMENTATION}

As discussed throughout this paper, there are several EIR program models that may be more or less suitable given the institutional context and desired outcomes. EIR programs can bring an industry perspective to curriculum development, the role of an instructor, mentors to clubs and teams, an as-needed resource to students, or even bring a workplace dynamic to campus. Additionally, the local industry landscape may also inform potential opportunities or barriers based on alignment, and dictate incentives and benefits related to engagement from industry partners. Careful consideration of these criteria from both sides may provide insight towards the best approach to establishing EIR programs.

A common challenge in Engineering Education is the devalued student perception of the importance of nontechnical professional skills (communication, teamwork, leadership, etc.). Contrary to faculty members who may not value or possess these skills, EIRs bring the relevant experience to convey the importance of these competencies. EIRs may serve as a unique gateway to meaningfully incorporating these non-technical skills within the curriculum.

Dalhousie mentioned the importance of including the department in the hiring process to ensure appropriate fit. Many institutions and departments have strong cultural identities, and considering EIRs are often appointed on limited terms or involvement, more quickly integrating into the community will likely maximize the value the EIR can provide.

Many EIRs take part in their roles coinciding with the later stages of their careers. It would be interesting to explore the effect of recruiting EIRs at the early stages of their careers, who may be able to relate to students on a more personal level having more recently graduated, and navigated the transition to the workplace - lens particularly relevant to students who will be following similar pathways. Finally, and unsurprisingly, a major topic throughout this work has been funding mechanisms. The innovative nature of EIR Programs also means that there are presently no obvious funding mechanisms, and positions are typically funded on term-limited soft money from industry and/or granting agencies. Sustainable 
funding mechanisms are a key part of sustainable EIR Programs that can plan for medium- and long-term impact.

\section{Acknowledgements}

The authors acknowledge the support of EIRs and those leading EIR programs at their respective universities, including those who lent time to interviews: Nika Zolfaghari, Gordana Andjelkovic, Deanna Burgart and Clifton Johnston for their time and their contribution to engineering education and the profession.

\section{References}

[1] Gordana Andjelkovic, EIR at University of Waterloo, Interview April 112019.

[2] John Bransford, Ann Brown, and Rodney Cocking, How People Learn: Brain, Mind, Experience, and School. Washington D.C.: National Academy Press, 1999.

[3] Deanna Burgart, EIR at Schulich School of Engineering, University of Calgary, Interview April 102019.

[4] Concordia University, "Wikipedian in Residence." Available as of April 6, 2019, from https://library.concordia.ca/about/jobs/wikipedian-inresidence.pdf

[5] Joshua Devitt, Shara Cameron, and Jim Morgan, "What is an Engineer in Residence?" in Proc. Australasian Association for Engineering Education Conf., AAEE2016, (Coffs Harbour, Australia: 4-7 December 2016), 7 pp., 2016.

[6] Barry Farbrother, and Michael Chow, "Paralleling the Workplace on the University Campus: The EiR Program at Ohio Northern University" in Proc. American Society for Engineering Education Conf, ASEE2003, (Nashville, TN: 2325 June 2003), 6 pp., 2003.

[7] Anthony Mann, Julian Stanley, and Louise Archer. (eds), "Chapter 2 - A theoretical framework for employer engagement" in Understanding Employer Engagement in Education: Theories and Evidence, London: Routledge (2014), pp.36-52.

[8] Alma Rosales, Andrea Leland, Olivera Notaros, Richard Toftness, Thomas Siller, Michael De Miranda, Alistair Cook, Melissa Reese, Zinta Byrne, James Warren Weston, and Anthony Maciejewski, "Preliminary Work on Weaving Professionalism Throughout the Engineering Curriculum" in Proc. American Society for Engineering Education Conf, ASEE2016, (New Orleans, LA: 26-29 June 2016), 19 pp., 2016.

[9] J.M. Symonds, and Ron Britton, "Engineers-in-Residence A Bridge to the Future" in Proc. CEEA Canadian Engineering Education Conf., CEEA12, (Winnipeg, MB ; 1720 June 2012), 3 pp., 2012.

[10] Ryerson University, Faculty of Engineering \& Architectural Science, "Stories March 9 2018." Available as of April 6, 2019, from https://www.ryerson.ca/engineeringarchitectural-science/stories-events/2018/03/hydro-onepowers-women-in-engineering/

[11] Ryerson University, The Ryerson Connection "Hydro One Powers Women in Engineering." Available as of April 6, 2019, from https://www.ryerson.ca/alumni/enewsletter/2018/11/featured hydro-one-powers-women-in-engineering/
[12] University of Calgary, Schulich School of Engineering, "Indigenous Strategy." Available as of April 6, 2019, from https://schulich.ucalgary.ca/about/outreachrecruitment/indigenous-strategy

[13] University of Manitoba, Asper School of Business, "Executives in Residence." Available as of April 6, 2019, from http://umanitoba.ca/faculties/management/executivesin-residence.html

[14] University of Manitoba, Centre for Engineering Professional Practice and Engineering Education, "Engineers in Residence." Available as of April 6, 2019, from http://umanitoba.ca/faculties/engineering/departments/ce2p2 e/Engineers-in-Residence.html

[15] University of Manitoba, Partners Program, "Partner-inResidence." Available as of April 6, 2019, from http://umanitoba.ca/faculties/architecture/partnersprogram/p artnerinresidence.html

[16] University of Manitoba, School of Art, "Indigeous Designer in Residence." Available as of April 6, 2019, from http://umanitoba.ca/schools/art/idir.html

[17] University of Ottawa, Civil Engineering. Available as of April 6, 2019, from https://www.letsgomoose.ca/wpcontent/uploads/MediaAdvisory_PrinceOfWalesBridge_UO ttawaEngineering_2018-10-17aaFINAL_PDF.pdf

[18] University of Ottawa, Faculty of Engineering, "Faculty Council Meeting, Feb 2018." Available as of April 6, 2019, from https://engineering.uottawa.ca/faculty-council-meeting$\underline{201802}$

[19] University of Ottawa, School of Electrical Engineering and Computer Science, "NSERC Chair in Entrepreneurial Engineering Design." Available as of April 6, 2019, from https://engineering.uottawa.ca/people/anis-hanan

[20] University of Toronto, Libraries, "U of T Libraries hires its first Wikipedian in Residence.” Available as of April 6, 2019, from https://onesearch.library.utoronto.ca/news/u-t-librarieshires-its-first-wikipedian-residence

[21] University of Toronto, Rotman Commerce, "Rotman Commerce welcomes first Executive-in-Residence." Available as of April 6, 2019, from https://rotmancommerce.utoronto.ca/article/rotmancommerce-welcomes-first-executive-residence-davidscrymgeour/

[22] University of Toronto, Rotman School of Management, "Executives in Residence." Available as of April 6, 2019, from

http://www.rotman.utoronto.ca/FacultyAndResearch/Faculty /ExecutivesInResidence

[23] University of Toronto at Scarborough, Department of English, "Minor in Creative Writing." Available as of April 6, 2019, from https://www.utsc.utoronto.ca/engdept/minorcreative-writing

[24] University of Waterloo, School of Planning, "Planner-inResidence." Available as of April 6, 2019, from https://uwaterloo.ca/planning/about/our-people/plannerresidence

[25] York University, Schulich School of Business, "Executives in Residence." Available as of April 6, 2019, from http://schulich.yorku.ca/about/our-dean/executives-inresidence/

[26] Nika Zolfaghari, Manager, Equity, Diversity and Inclusion in the Faculty of Engineering and Architectural Science at Ryerson University. Interview Feb 42019. 